\title{
Die Änderungen im Zugewinnausgleichsrecht
}

Das Bundesministerium der Justiz bat den djb um eine Stellungnahme, zum Gesetzentwurf zur Änderung des Zugewinnausgleichsrechts. Diese Gelegenheit nahmen wir - wie immer - gerne wahr.

Grundsätzlich sind die geplanten Neuerungen zu begrüßen, denn sie stellen insgesamt eine Verbesserung dar. Da die Zugewinngemeinschaft als gesetzlicher Güterstand der mehrheitlich gelebte Güterstand in Deutschland ist, verdient er natürlich besondere Aufmerksamkeit und bedarf bevorzugt der Korrektur seiner Schwächen. Dies gilt umso mehr, als nach der neueren höchstrichterlichen Rechtssprechung der Gestaltungsmöglichkeit durch Eheverträge Grenzen gezogen werden, so dass der gesetzliche Güterstand noch größere praktische Bedeutung gewinnt und seine Ausgewogenheit für noch mehr Ehen wichtig ist.

Ein Manko des bisherigen Rechts war insbesondere, dass Schulden im Anfangs- und im Endvermögen keine Berücksichtigung fanden. ${ }^{1}$

Das Schuldenprivileg des $\mathbb{S} 1374$ BGB führte dazu, dass der Ehepartner, der sich an der Rückzahlung der in die Ehe mitgebrachten Schulden des Partners beteiligte, beim Scheitern der Ehe insofern dafür bestraft wurde, als die Schuldentilgung unberücksichtigt blieb. Kam also zum Beispiel der Mann mit 100.000 Euro Schulden in die Ehe und hatte am Ende der Ehe nur noch 10.000 Euro Schulden, so hatte die Frau nichts davon, dass sich der Schuldenstand verringert hatte. Im Gegenteil, sofern sie selbst einen Zugewinn erzielt hätte, z.B. 40.000 Euro, hätte sie davon 20.000 Euro abgeben müssen, so dass der Partner letztlich einen wirtschaftlichen Gewinn von 110.000 Euro (90.000 Euro Schuldentilgung plus 20.000 Euro Zugewinnausgleichsanspruch) machte.

Derartige Ergebnisse werden vermieden, wenn die Schulden in die Bilanz des Anfangsvermögens aufgenommen werden und auch im Endvermögen über die Höhe der Aktiva hinaus zu berücksichtigen sind.

Der grundsätzliche Ansatz ist also richtig.

Aber Unausgewogenheiten bestehen fort.

Die neue Fassung des Gesetzes führt nämlich in den Fällen zu keinem den Halbteilungsgrundsatz wahrenden Ergebnis, in denen der verschuldete Ehegatte auch am Ende der Ehe noch verschuldet ist und der andere Zugewinn erworben hat. Denn auch das neue Recht sieht in $\$ 1378$ Abs. 2 BGB-E eine Kappungsgrenze vor, und zwar auf die Hälfte des vorhandenen Vermögens. In obigem Beispiel könnte die Frau also nichts verlangen, weil kein Aktivvermögen da ist.
Sie könnte ihren Anspruch von z.B. 30.000 Euro aber selbst dann nicht voll durchsetzen, wenn der Mann ein Endvermögen von 20.000 Euro hätte; dann bekäme sie nur die Hälfte davon, also 10.000 Euro. Auch das kann nicht befriedigen.

Die Bestimmung des $\mathbb{} 1378$ II BGB-E ist also nicht akzeptabel. Sie würde gegenüber der derzeitigen Situation sogar eine erheblichere Benachteiligung des Ausgleichsberechtigten bewirken. Denn jetzt ist es so, dass der Anspruch auf die gesamte Höhe des Endvermögens begrenzt ist und nicht nur auf die Hälfte. ${ }^{2}$

Zwar ist es einzusehen, dass der Pflichtige keine Schulden machen soll; er soll sich aber auch nicht zu Lasten der oder des Berechtigten auf den Halbteilungsgrundsatz berufen dürfen - während gerade für den anderen der Halbteilungsgrundsatz nicht gilt.

Die Auskunftspflichten wurden erweitert. Auch über das Anfangsvermögen muss künftig Auskunft erteilt werden, nicht nur wie bisher über das Endvermögen. ${ }^{3}$ Diese Erweiterung ist erforderlich, da bei der Berechnung des Ausgleichsanspruches nunmehr Schulden zu Beginn der Ehe berücksichtigt werden und deren Höhe erfassbar sein muss.

Begrüßenswert ist, dass die Vorlage von Belegen für End- und Anfangsvermögen zur Pflicht wird. ${ }^{4}$ Die bisherige Rechtslage war für die Praxis ungut, weil es keinen Anspruch gab, Belege einzufordern, wie es anders das Unterhaltsrecht (\$ 1605 i.V.m. \$1590) schon kannte.

Für die Berechnung des Zugewinnausgleichsanspruchs und die Höhe der Ausgleichsforderung wird es einen einheitlichen Stichtag geben, nämlich den Tag der Rechtshängigkeit des Scheidungsantrags. ${ }^{5}$ Vermögensminderungen während des Scheidungsverfahrens werden also unbeachtlich sein. Damit folgt der Entwurf einer seit langem im Schrifttum erhobenen Kritik an der bisherigen Regelung, die Manipulationen zu Lasten des Ehepartners während dieser Zeit möglich machten. ${ }^{6}$ Die derzeitige Kappungsgrenze des $\mathbb{} 1378$ II BGB (Begrenzung des Ausgleichsanspruchs auf das bei Scheidung vorhandene Vermögen) wird also entfallen.

Weiter wird es einen Schutz vor illoyalen Vermögensminderungen durch $\mathbb{} 1378$ Abs. 2 BGB-E und $\mathbb{1 3 9 0}$ BGB-E geben.

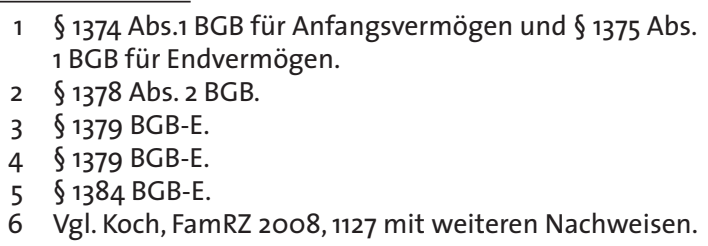

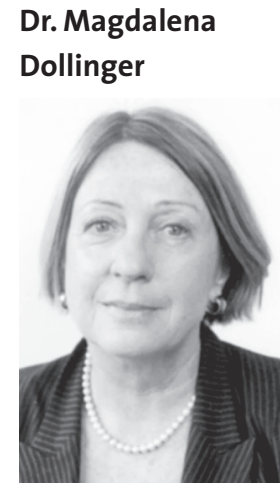

Mitglied der Kommission Zivil-, Familienund Erbrecht, Recht anderer Lebensgemeinschaften des djb, Rechtsanwältin, Fachanwältin für Familienrecht, München 
In $\ 1378$ Abs. 2 BGB-E ist vorgesehen, dass die Ausgleichsforderung nicht begrenzt wird, wenn sie wegen fiktiver Hinzurechnung verausgabter Summen die Hälfte des vorhandenen Vermögens übersteigt. Der unredliche Ehegatte muss dann sein gesamtes Vermögen hergeben und sich sogar verschulden, um die Zugewinnausgleichsforderung zu erfüllen. ${ }^{7}$ Der unredliche Ehegatte soll nicht geschützt werden und nicht im Besitz der „verschwundenen“ Habe bleiben.

\ 1390 BGB-E enthält einen Herausgabeanspruch gegen Dritte, die Empfänger der illoyalen Vermögensminderung sind, wenn die Ausgleichsforderung das vorhandene Vermögen des Ehegatten übersteigt; die Voraussetzungen des Herausgabeanspruches zu der bisherigen Bestimmung ändern sich. ${ }^{8}$

Allerdings dürfte auch mit diesen Änderungen nicht die Gefahr beseitigt sein, dass alle illoyalen Vermögensverschiebungen verhindert bzw. ausgeglichen werden. Denn das praktische Problem ist, dass solche Manipulationen häufig schwer nachzuvollziehen und zu beweisen sind. Erwägenswert wäre, eine generelle Auskunftspflicht für die gesamte Dauer der Ehe einzurichten.

Viele Ehepaare haben eine Aufgabenverteilung dergestalt, dass sich der eine um die Finanzen kümmert - oft der Mann - und die Frau keinerlei Interesse und Einblick in diese Materie hat. Das mag gut sein für gute Zeiten; wenn es aber um die Auseinandersetzung geht, dann sollten beide berechtigt sein zu erfahren, was im Laufe der Ehe mit den gemeinsamen Geldern und dem Vermögen passiert ist. Aus dem Grundsatz der ehelichen Solidarität lässt sich ein solcher Anspruch zwanglos herleiten.

Der Entwurf befasst sich nicht damit, dass Wertsteigerungen des Anfangsvermögens oder privilegierten Erwerbs ausgleichspflichtig sind.

Es ist eine Frage der Position, ob man es als gerecht empfindet, dass nach gescheiterter Ehe ein Partner an Vermögenszu- wächsen teilhat, die nichts mit einer gemeinsamen Leistung zu tun haben, die während der Ehe erbracht wurde. Wenn die Eheleute zusammenbleiben, sollen beiden derartige Erträge sicherlich gebühren. Ob jedoch ein Anspruch auf derartige Werte auch einem Ehepartner/einer Ehepartnerin zusteht, wenn er oder sie aus der Ehe ausbricht, ist meines Erachtens nicht unbedingt einzusehen bzw. zumindest diskutabel.

Im Vorfeld unserer Stellungnahme haben wir diskutiert, ob nicht generell der Errungenschaftsgemeinschaft als gesetzlichem Güterstand der Vorzug einzuräumen sei. ${ }^{9}$

Die Zugewinngemeinschaft findet ihresgleichen im europäischen Familienrecht nicht. Der häufigste Güterstand in Europa ist die Errungenschaftsgemeinschaft, die ja auch in Deutschland bis zur Mitte des letzten Jahrhunderts einer der gesetzlichen Güterstände war. Es stellt sich die Frage, ob die Zugewinngemeinschaft als gesetzlicher Güterstand nicht nach wie vor Schwächen aufweist, die die Errungenschaftsgemeinschaft vorzugswürdig erscheinen lassen. Hingewiesen sei auf die vorstehend erwähnten Manipulationsmöglichkeiten oder etwa auf die ausgleichspflichtigen Wertzuwächse, die ohne Leistung des Anspruchsberechtigen bestehen. Auch ist in der Praxis oftmals das System der Zugewinngemeinschaft Laien schwer zu vermitteln; das reine Stichtagsprinzip ohne ausreichende Möglichkeit der Bereinigung zu Ehezeiten erfolgter Vermögensverschiebungen ist Laien vielfach nicht als gerecht darzustellen. Die jetzige Rechtsänderung dürfte innerhalb der europäischen Entwicklung nicht das letzte Wort zum ehelichen Güterrecht gewesen sein.

\footnotetext{
7 Koch, a.a.O.

8 Näheres hierzu vgl. Koch, a.a.O., S. 1129.

9 Vgl. dazu auch, ob die Reformen im Familienrecht Kosmetik oder Kurskorrektur sein sollen, Born in NJW 2008, 2289 ff.
}

\section{Gesetz zur Strukturreform des Versorgungsausgleichs}

\section{Der lange Weg zu mehr Gerechtigkeit?}

\section{Stand des Gesetzgebungsverfahrens}

Nachdem sich die vom Bundesministerium der Justiz im Jahr 2003 eingesetzte Kommission in ihrem Abschlussbericht nicht auf eine umfassende Neuordnung und Neubewertung der Versorgungssysteme in allen ihren Facetten verständigen konnte, ist vor dem Hintergrund mahnender Entscheidungen aus Karlsruhe eine Reform unumgänglich. ${ }^{1}$

Der Diskussionsentwurf vom 29. August 2007, der in seinen Ausgleichsformen nahezu unverändert dem Referentenentwurf vom 12. Februar 2008 (und auch dem Regierungsentwurf) entspricht, trifft überwiegend auf Zustimmung.

Bei näherer Betrachtung kommen indes Zweifel auf, ob der konzeptionelle Ansatz - Vorrang der internen Teilung - die Probleme des Versorgungsausgleichs in seiner geltenden Fassung überzeugend löst.
Die aus der Praxis vorgebrachte Kritik ${ }^{2}$ hat bislang nicht zu Änderungen oder Nachbesserungen des Entwurfs geführt; ob sich dieses im laufenden Gesetzgebungsverfahren ändern wird, bleibt abzuwarten.

\section{Die Zielsetzung: Interne Teilung der Anrechte}

Nach geltendem Recht erfolgt der Ausgleich der Versorgungsanwartschaften - dies darf vorausgeschickt werden - nach Saldierung aller Anrechte. Derjenige Ehepartner, der die werthöheren Anwartschaften erworben hat, ist in Höhe des hälftigen Wertunterschiedes ausgleichsverpflichtet, wobei - von ei-

\footnotetext{
1 BGH FamRZ 01, 1695 = NJW 02, 296 ff.; BVerfG NJW o6, 2177ff.)

2 (Rehme FamRZ 08, 738 ff. zu den verfassungsrechtlichen Bedenken; Born NJW 08, 2289 ff. zum „niedrigen Gerechtigkeitsniveau“.
} 